\title{
NON-CONSTANT POSITIVE SOLUTIONS OF A GENERAL GAUSE-TYPE PREDATOR-PREY SYSTEM WITH SELF- AND CROSS-DIFFUSIONS*
}

\author{
Pan Xue ${ }^{1}$, Yunfeng Jia ${ }^{2, * *}$, Cuiping Ren ${ }^{1}$ And Xinguun Li $^{1}$
}

\begin{abstract}
In this paper, we investigate the non-constant stationary solutions of a general Gause-type predator-prey system with self- and cross-diffusions subject to the homogeneous Neumann boundary condition. In the system, the cross-diffusions are introduced in such a way that the prey runs away from the predator, while the predator moves away from a large group of preys. Firstly, we establish $a$ priori estimate for the positive solutions. Secondly, we study the non-existence results of non-constant positive solutions. Finally, we consider the existence of non-constant positive solutions and discuss the Turing instability of the positive constant solution.
\end{abstract}

Mathematics Subject Classification. 35K51, 35K57, 35A01, 35A09.

Received August 16, 2020. Accepted March 22, 2021.

\section{INTRODUCTION}

In this paper, we investigate the existence and non-existence of the non-constant positive stationary solutions for the following general Gause-type predator-prey system with constant self- and cross-diffusions

$$
\begin{cases}u_{t}-d_{1} \Delta\left[\left(1+d_{3} v\right) u\right]=u g(u)-p(u) v, & (x, t) \in \Omega \times(0, \infty), \\ v_{t}-d_{2} \Delta\left[\left(1+d_{4} u\right) v\right]=v(-d+c p(u)), & (x, t) \in \Omega \times(0, \infty), \\ u(x, 0)=u_{0}(x) \geq 0, v(x, 0)=v_{0}(x) \geq 0, & x \in \Omega, \\ \partial_{\nu} u=\partial_{\nu} v=0, & (x, t) \in \partial \Omega \times(0, \infty),\end{cases}
$$

where $\Omega \subset R^{N}$ ( $N \geq 1$ be an integer) is a bounded domain with smooth boundary $\partial \Omega, \nu$ is the outward unit normal vector on the boundary $\partial \Omega$ with $\partial_{\nu}=\frac{\partial}{\partial \nu}$, and the homogeneous Neumann boundary condition means that the individuals do not cross the habitat boundary, the variables $u$ and $v$ represent the densities of prey and predator, respectively, the coefficient $c>0$ refers to the conversion rate of prey captured by the predator and $d>0$ represents the predator's death rate.

* This work is supported in part by the National Natural Science Foundations of China (No. 11771262), by the Natural Science Research Program of Shaanxi Provincial Department of Education (No. 20JK0815), and also by the Science Research Program of Xi'an Eurasia University (No. 2020XJZK10).

Keywords and phrases: Cross-diffusion; predator-prey system; positive solutions; Leray-Schauder degree; stability.

1 School of General Education, Xi'an Eurasia University, Xi'an, Shaanxi 710065, China.

2 School of Mathematics and Statistics, Shaanxi Normal University, Xi'an, Shaanxi 710062, China.

** Corresponding author: jiayf@snnu.edu.cn

(C) The authors. Published by EDP Sciences, 2021

This is an Open Access article distributed under the terms of the Creative Commons Attribution License (https://creativecommons.org/licenses/by/4.0), which permits unrestricted use, distribution, and reproduction in any medium, provided the original work is properly cited. 
Throughout this paper, the function $g(u) \in C^{1}([0,+\infty))$ describes the growth rate of prey when the predator is absent, i.e., the intrinsic growth rare of prey and it also measures the availability of using the natural resources. The function $p(u) \in C^{1}([0,+\infty)) \cap C^{2}((0,+\infty))$ represents the consumption rate of prey by predator as a result of a change in the prey density. In order to describe the biological significance of the system, $g(u)$ and $p(u)$ are assumed to be satisfying the following two assumptions.

(A1) $g(0)>0$ and $g^{\prime}(u)<0$ hold for all $u \geq 0$ and there exists a unique positive constant $S$, such that $g(S)=0$; (A2) $p(0)=0, \lim _{u \rightarrow 0^{+}} p^{\prime}(u)<\infty, c p(S)>d$, and $p^{\prime}(u)>0$ for all $u \in[0, S)$.

As some examples of the function $g(u)$ satisfying the assumption (A1), the following two concrete types can be introduced.

Type 1: $g(u)=\frac{r(K-u)}{K+\epsilon u}$;

Type 2: $g(u)=r\left(1-\left(\frac{u}{K}\right)^{\theta}\right)$,

where the coefficients $r, K$ and $\theta \geq 1$ are all positive constants, whereas the coefficient $\epsilon$ is non-negative constant. According to [4], type 2 is the classical $\theta$-logistic growth rate.

In fact, the growth rate of the predator is improved in the presence of prey by an amount proportional to the number of prey. Therefore, the functional response $p(u)$ can be explained as the consumption rate at which the prey is consumed by an individual predator. In terms of the functional response $p(u)$, it can be divided into many forms, for instance, the classical Holling-type functional responses (see [1, 6, 20-22] for examples), the Ivlev and the inverted Ivlev functional responses, and so on (see $[5,23]$ for examples). We note that among these functional responses, the Holling I, II and the Ivlev types all satisfy the assumption (A2). Meanwhile, we point out that system (1.1) with assumption (A2) does not contain the systems with non-monotonic functional responses, such as $p(u)=\frac{u}{a+u^{2}}$ with $a$ being a positive constant.

In system (1.1), the coefficients $d_{1}$ and $d_{2}$ are both positive constants and $d_{3}, d_{4}$ are non-negative constants. In our system, the self- and cross-diffusions satisfy the following two identities,

$$
-d_{1} \Delta\left[\left(1+d_{3} v\right) u\right]=-\operatorname{div}\left\{\nabla\left(d_{1} u+d_{1} d_{3} u v\right)\right\}, \quad-d_{2} \Delta\left[\left(1+d_{4} v\right) u\right]=-\operatorname{div}\left\{\nabla\left(d_{2} v+d_{2} d_{4} u v\right)\right\} .
$$

The diffusion terms $d_{i}, i=1,2$ stand for the natural dispersive force of movement of an individual prey or predator. While the cross-diffusions pressure $d_{3}$ and $d_{4}$ (or $d_{1} d_{3}$ and $d_{2} d_{4}$ ), which can not be described in classical predator-prey systems, plays an important role in generating various spatial patterns in biological population dynamics (see $[12,18]$ ).

Precisely speaking, we regard the term $d_{1} u+d_{1} d_{3} u v$ as the survival conditions of prey, meaning the diffusion is toward the places of better survival conditions. The diffusion $d_{1} \Delta\left[\left(1+d_{3} v\right) u\right]$ of population can be explained as follows. Denoting $\Delta u=\operatorname{div}(\nabla u)$, we may consider $\nabla u$ as the flux. Then, the prey $u$ diffuses with flux

$$
-\nabla\left(d_{1} u+d_{1} d_{3} u v\right)=-d_{1} d_{3} u \nabla v-\left(d_{1}+d_{1} d_{3} v\right) \nabla u .
$$

We perceive that, as $d_{1} d_{3} u>0$, the part $-d_{1} d_{3} u \nabla v$ of flux is directed toward the decreasing population density of the predator. Particularly, when $d_{3}$ is large, the term $-d_{1} d_{3} u \nabla v$ represents the preys running away from a large group of predators.

Similarly, the predator $v$ diffuses with flux

$$
-\nabla\left(d_{2} v+d_{2} d_{4} u v\right)=-d_{2} d_{4} v \nabla u-\left(d_{2}+d_{2} d_{4} u\right) \nabla v
$$

We notice that, since $d_{2} d_{4} v \geq 0$, the part $-d_{2} d_{4} v \nabla u$ of flux is directed toward the decreasing population density of the prey. Particularly, when the diffusion term $d_{4}$ is large enough, $-d_{2} d_{4} v \nabla u$ represents the predator far away from a large group of preys. Generally speaking, if a prey species has a pretty high populations density or size, it is usually difficult for other prey or predator species to invade its habitat. For more information and 
background, see $[7,13,16,24]$ for detailed discussion and explanation on relevant and homologous biological systems.

Over the past few years, there are many valuable results and progresses on the effects of self-diffusion and cross-diffusion on the dynamics of different systems. In mathematical biological ecology, the classical predatorprey system, independently, introduced by Lotka and Volterra in the 1920s, only reflects the population changes caused by predation when the density of predator and prey is not spatially dependent. It usually does not consider the fact that the population distribution is uneven, nor does it consider the fact that predator and prey naturally form survival strategies.

Both of these considerations involve diffusion processes, which can explain that different concentrations of predators and preys can bring about different movements. These movements can be determined by the concentration (diffusion) of the same species and the concentration (cross-diffusion) of other species. Additionally to the classical diffusion, self- and cross-diffusions allow to take into account the effect of pressure between species. For example, at a point where the term $u v$ is at local maximum, $\Delta u$ is negative, which stands for the fact that the prey tends to run away from there. Roughly speaking, the population growth systems including cross-diffusions can produce more complex and abundant population dynamics than the population growth model without considering these factors.

The role of self- and cross-diffusions in modeling of various biological, physical, and chemical processes has been widely investigated. For example, in $[2,15]$, the authors investigated the existence of steady state in a linear cross-diffusion predator-prey system with Beddington-DeAngelis functional response and the proofs mainly depend on analytical techniques and fixed point index theory.

Paper [25] considered a strongly coupled predator-prey system arising from the classical Lotke-Volterra system, in which the cross-diffusions are included in such a way that the predator moves away from a large group of preys and the prey flees away from the predator. The author established the non-existence and existence of its non-constant positive solutions for suitable large values of the cross-diffusion or small enough cross coefficients. In [9], without taking into account the cross-diffusions, the authors studied the qualitative behavior of non-constant positive solutions on a general Gause-type predator-prey system subject to the homogeneous Neumann boundary condition, in which with diffusion rates are constants. The results show that the non-existence and existence of the non-constant positive steady-state solutions are affected by the self- and cross-diffusion rates. In addition, they also investigated the local existence of periodic solutions, the asymptotic behavior of spatially inhomogeneous solutions, and the diffusion-driven instability. Recently, a special case of system (1.1) was considered in [8], specifically, the cross-diffusion rate $d_{4}$ is absent, in which, the cross-diffusion $d_{1} d_{3} u$ is included in the meaning of the prey escapes from the predator. The paper investigated the existence of nonconstant positive steady states and discuss the stability of constant equilibrium point. And the result points out the conditions which can provide the system admits a non-constant positive steady state. In fact, there also have abundant and important results and conclusions on the mathematical developments of self- and cross-diffusion systems produced in various research fields, see [14, 17], for example.

As far as we know, there are few works related system (1.1) with the cross-diffusions for both predator and prey. Inspired by these works, we investigate the existence of the non-constant positive solutions of system (1.1) by using the well-known Leray-Schauder degree theory. We justify the choice of nonlinear reaction terms by referring to the classical expression in use in literature and usual references. Since the cross-diffusion terms $d_{3}$ and $d_{4}$ are introduced, the corresponding computation and analysis in the subsequence are more difficult and require a bit of techniques than systems without considering the cross-diffusions term. The main aim of this paper is to investigate the effect of the self- and cross-diffusions pressures on the non-constant positive solutions of system (1.1), that is, we study the non-existence and the existence of the non-constant positive solutions to the following elliptic system

$$
\begin{cases}-d_{1} \Delta\left[\left(1+d_{3} v\right) u\right]=u g(u)-p(u) v, & x \in \Omega, \\ -d_{2} \Delta\left[\left(1+d_{4} u\right) v\right]=v(-d+c p(u)), & x \in \Omega, \\ \partial_{\nu} u=\partial_{\nu} v=0, & x \in \partial \Omega\end{cases}
$$


The organization of this paper is as follows. In Section 2, we establish a priori estimate for positive solutions of the system (1.2) by employing the Harnack inequality and the maximum principle. In Section 3, we study the non-existence results of the non-constant positive solutions by using the energy integral method. We mainly consider the influence of the change of diffusion coefficients on the existence of non-constant positive solutions. In Section 4, we mainly consider the existence of the non-constant positive solutions by making use of the LeraySchauder degree theory. Moreover, we also discuss the Turing instability of system (1.1) mainly by considering the influence of the diffusion terms.

\section{The PRIORI ESTIMATE OF NON-CONSTANT POSITIVE SOLUTIONS}

The main purpose of this section is to establish the priori upper and lower estimates with respect to the diffusion coefficients $d_{i}$ for the non-constant positive solutions of system (1.2). In order to guarantee the result, we first give a brief account of the following two preliminaries, that is, the Harnack inequality and the Maximum principle. These two results are usually used to prove the priori estimates of positive solutions.

Lemma 2.1. [11] Assume that $c(x) \in C(\bar{\Omega})$ and let $\omega(x) \in C^{2}(\Omega) \cap C^{1}(\bar{\Omega})$ be a positive solution to

$$
\Delta \omega(x)+c(x) \omega(x)=0, \quad x \in \Omega, \quad \partial_{\nu} \omega=0, \quad x \in \partial \Omega .
$$

Then there exists a positive constant $C^{*}=C^{*}\left(\|c\|_{\infty}, \Omega\right)$, such that $\max _{\bar{\Omega}} \omega \leq C^{*} \min _{\bar{\Omega}} \omega$.

Lemma 2.2. [13] Let $\phi(u, x) \in C\left(\Omega \times R^{1}\right)$. If $u \in C^{2}(\Omega) \cap C^{1}(\bar{\Omega})$ satisfies $\Delta u(x)+\phi(u, x) \geq 0$ in $\Omega$, $\partial_{\nu} u=0$ on $\partial \Omega$ and achieves its maximum at a point $x_{0} \in \bar{\Omega}$, then $-\Delta u\left(x_{0}\right) \geq 0$.

Theorem 2.3. Let $D_{1}, D_{2}, D_{3}, D_{4}$ be given positive constants. Then there exists a positive constant $\bar{C}=$ $\bar{C}\left(c, d, D_{1}, D_{2}, D_{3}, D_{4}, \Omega\right)$ which is independent of $u$ and $v$, such that every positive solution $(u, v)$ of system (1.2), satisfies $u(x), v(x)<\bar{C}$ for $d_{2} \geq D_{2}, d_{3} \leq D_{3}$ and $d_{4} \leq D_{4}$.

Proof. Suppose that $(u, v)$ be a positive solution of system (1.2). We denote $\phi=\left(1+d_{3} v\right) u, \varphi=\left(1+d_{4} u\right) v$ and then system (1.2) can be rewritten as

$$
\begin{cases}-d_{1} \Delta \phi=u g(u)-p(u) v, & x \in \Omega, \\ -d_{2} \Delta \varphi=v(-d+c p(u)), & x \in \Omega, \\ \partial_{\nu} u=\partial_{\nu} v=0, & x \in \partial \Omega .\end{cases}
$$

Let $x_{1} \in \bar{\Omega}$ be a point satisfying $\phi\left(x_{1}\right)=\max _{\bar{\Omega}} \phi(x)$ and apply the Maximum principle to the first equation of system (2.1), we obtain

$$
u\left(x_{1}\right) g\left(u\left(x_{1}\right)\right)-p\left(u\left(x_{1}\right)\right) v\left(x_{1}\right) \geq 0 .
$$

Hence, we get $u\left(x_{1}\right) g\left(u\left(x_{1}\right)\right) \geq 0$. By the assumptions (A1) and (A2), we have $u\left(x_{1}\right)>0, p\left(u\left(x_{1}\right)\right)>0$. Thus,

$$
0<v\left(x_{1}\right) \leq \frac{u\left(x_{1}\right) g\left(u\left(x_{1}\right)\right)}{p\left(u\left(x_{1}\right)\right)} \leq \frac{g(0) u\left(x_{1}\right)}{p\left(u\left(x_{1}\right)\right)} .
$$

Simple mathematical analysis shows that $0<\inf _{u \in(0, S)} \frac{u}{p(u)} \leq \sup _{u \in(0, S)} \frac{u}{p(u)}<\infty$ by the assumption (A2) and $p(u) \in C^{1}([0,+\infty)) \cap C^{2}((0,+\infty))$, even if $p(u) \rightarrow 0$. This indicates that $\frac{g(0) u\left(x_{1}\right)}{p\left(u\left(x_{1}\right)\right)}$ is bounded, we denote an upper bound by $M$. Thus, there holds

$$
\max _{\bar{\Omega}} u(x) \leq \max _{\bar{\Omega}} \phi(x)=\left(1+d_{3} v\left(x_{1}\right)\right) u\left(x_{1}\right) \leq\left(1+D_{3} M\right) S \triangleq C_{1} .
$$


We multiply the first equation of system (1.2) by constant $c$, then add it to the other equation. Finally, we integrate it over $\Omega$ and we can achieve

$$
\int_{\Omega}\left\{-c d_{1} \Delta\left[\left(1+d_{3} v\right) u\right]-d_{2} \Delta\left[\left(1+d_{4} u\right) v\right]\right\} \mathrm{d} x=\int_{\Omega}[-\mathrm{d} v+c u g(u)] \mathrm{d} x .
$$

According to the divergence theorem, we obtain

$$
\int_{\Omega}\left\{-c d_{1} \Delta\left[\left(1+d_{3} v\right) u\right]-d_{2} \Delta\left[\left(1+d_{4} u\right) v\right]\right\} \mathrm{d} x=0 .
$$

Thus, we get

$$
d \int_{\Omega} v \mathrm{~d} x=\int_{\Omega} \operatorname{cug}(u) \mathrm{d} x \leq \int_{\Omega} c g(0) C_{1} \mathrm{~d} x=c g(0) C_{1}|\Omega|
$$

that is

$$
\int_{\Omega} v \mathrm{~d} x \leq \frac{c g(0) C_{1}|\Omega|}{d} .
$$

Also, we notice that system (1.2) can also be rewritten as

$$
\begin{cases}-\Delta \phi=\frac{g(u)-\frac{p(u) v}{u}}{d_{1}\left(1+d_{3} v\right)} \phi, & x \in \Omega \\ -\Delta \varphi=\frac{-d+c p(u)}{d_{2}\left(1+d_{4} u\right)} \varphi, & x \in \Omega \\ \partial_{\nu} \phi=\partial_{\nu} \varphi=0, & x \in \partial \Omega\end{cases}
$$

Since $\left\|\frac{-d+c p(u)}{d_{2}\left(1+d_{4} u\right)}\right\|_{\infty} \leq\left\|\frac{-d+c p(u)}{d_{2}}\right\|_{\infty} \leq\left\|\frac{d+c p(S)}{D_{2}}\right\|_{\infty}<\infty$ for $d_{2} \geq D_{2}$, the Harnack inequality is valid for $\varphi$, thus,

$$
\max _{\bar{\Omega}} \varphi \leq C_{0}^{*} \min _{\bar{\Omega}} \varphi
$$

for some suitable large positive constant $C_{0}^{*}$. Thus, we can obtain

$$
\max _{\bar{\Omega}} v \leq \max _{\bar{\Omega}} \varphi \leq C_{0}^{*} \min _{\bar{\Omega}} \varphi=C_{0}^{*} \min _{\bar{\Omega}}\left(1+d_{4} u\right) v \leq \frac{\left(1+D_{4} C_{1}\right) C_{0}^{*} \int_{\Omega} v \mathrm{~d} x}{|\Omega|} \leq \frac{\left(1+D_{4} C_{1}\right) c g(0) C_{1} C_{0}^{*}}{d} \triangleq C_{2} .
$$

Denote $\bar{C}=\max \left\{C_{1}, C_{2}\right\}$. Then the result follows.

Theorem 2.4. Let $D_{1}, D_{2}, D_{3}, D_{4}$ be given positive constants. Then there exists a positive constant $\underline{C}=$ $\underline{C}\left(c, d, D_{1}, D_{2}, D_{3}, D_{4}, \Omega\right)$ which is independent of $u$ and $v$, such that every positive solution $(u, v)$ of system (1.2), satisfies $\underline{C}<u(x), v(x)$ for $d_{1} \geq D_{1}, d_{2} \geq D_{2}, d_{3} \leq D_{3}$ and $d_{4} \leq D_{4}$.

Proof. We integrate the first equation of system (1.2) over $\Omega$. Then we get

$$
\int_{\Omega}[u g(u)-p(u) v] \mathrm{d} x=0 .
$$

Therefore, there must exist a point $x_{2} \in \Omega$ such that

$$
u\left(x_{2}\right) g\left(u\left(x_{2}\right)\right)-p\left(u\left(x_{2}\right)\right) v\left(x_{2}\right)=0 .
$$


By the assumptions (A1) and (A2), we get $0<u\left(x_{2}\right)<S$. Hence $v\left(x_{2}\right)=\frac{u\left(x_{2}\right) g\left(u\left(x_{2}\right)\right)}{p\left(u\left(x_{2}\right)\right)}>0$. If $\min _{\bar{\Omega}} v \rightarrow 0$, then inequality (2.4) implies that $\max _{\bar{\Omega}} v \rightarrow 0$. We get a contradiction, then $v$ has a positive lower bound when $d_{2} \geq D_{2}$.

Next, we mainly prove that $u$ also has a positive lower bound.

According to assumption (A2), $p(u) \in C([0,+\infty)) \cap C^{2}((0,+\infty))$ and $\lim _{u \rightarrow 0^{+}} \frac{p(u)}{u}=\lim _{u \rightarrow 0^{+}} p^{\prime}(u)<\infty$, we can assert that there must exist a positive constant $\hat{p}$ such that $\frac{p(u)}{u} \leq \hat{p}$ for some small $u, 0<u \leq S$. Obviously, we obtain

$$
\left\|\frac{g(u)-\frac{p(u) v}{u}}{d_{1}\left(1+d_{3} v\right)}\right\|_{\infty} \leq\left\|\frac{g(0)+\hat{p} C_{2}}{D_{1}}\right\|_{\infty}<\infty
$$

when $d_{1} \geq D_{1}$. Hence, the Harnack inequality holds for $\phi$, namely

$$
\max _{\bar{\Omega}} \phi \leq C_{1}^{*} \min _{\bar{\Omega}} \phi
$$

for some large positive constant $C_{1}^{*}$. Thus, we have

$$
\frac{\max _{\bar{\Omega}} u}{\min _{\bar{\Omega}} u} \leq \frac{\max _{\bar{\Omega}} \phi}{\min _{\bar{\Omega}} \phi} \cdot \frac{1+d_{3} \max _{\bar{\Omega}} v}{1+d_{3} \min _{\bar{\Omega}} v} \leq C_{1}^{*}\left(1+d_{3} \max _{\bar{\Omega}} v\right) \leq C_{1}^{*}\left(1+D_{3} C_{2}\right) \triangleq C_{2}^{*} .
$$

On the other hand, assume that there exists a sequence $\left\{\left(d_{1 n}, d_{2 n}, d_{3 n}, d_{4 n}\right)\right\}, n=1,2, \cdots$, satisfying $d_{1 n} \geq$ $D_{1}, d_{2 n} \geq D_{2}, d_{3 n} \leq D_{3}$ for some $D_{3}>0$, and $d_{4 n} \leq D_{4}$, such that the corresponding positive solutions $\left(u_{n}, v_{n}\right)$ of system (1.2) with $\left(d_{1}, d_{2}, d_{3}, d_{4}\right)=\left(d_{1 n}, d_{2 n}, d_{3 n}, d_{4 n}\right)$ satisfy $\min _{\bar{\Omega}} u_{n} \rightarrow 0$ as $n \rightarrow \infty$. Combining with (2.6), we have $\max _{\bar{\Omega}} u_{n} \rightarrow 0$ as $n \rightarrow \infty$. By the regularity theory for elliptic equations [3], we have $u_{n} \in C^{2, \alpha}(\Omega)$. Therefore, we know that there exists a subsequence of $\left\{\left(u_{n}, v_{n}\right)\right\}$, which will be also denoted by $\left\{\left(u_{n}, v_{n}\right)\right\}$, such that $u_{n} \rightarrow 0$ converges uniformly as $n \rightarrow \infty$. We integrate the second equation of system (1.2) with $(u, v)=\left(u_{n}, v_{n}\right)$ and we can obtain

$$
\int_{\Omega} v_{n}\left(-d+c p\left(u_{n}\right)\right) \mathrm{d} x=0
$$

Since $u_{n} \rightarrow 0$ as $n \rightarrow \infty$, we have $-d+c p\left(u_{n}\right)<0$ on $\bar{\Omega}$ for any large enough integer $n$. This contradicts to the identity $(2.7)$ by considering $v_{n}>0$. In summary, there is $\underline{C}$ such that $\underline{C}<u(x), v(x)$. This finishes the proof.

\section{Non-EXISTEnCE OF THE NON-CONSTANT POSITIVE SOLUTIONS}

In this section, we analysis the results of non-existence of the non-constant positive solution of system (1.2) mainly by using the well-known Poincaré inequality and the energy integral method. We mainly consider the influence of the change of diffusion coefficients on the existence of non-constant positive solutions. To this end, we first introduce the following notations.

Let $\mu_{1}$ be the smallest positive eigenvalue of the operator $-\Delta$ subject to the homogeneous Neumann boundary condition. Denote

$$
\tilde{u}=\frac{1}{|\Omega|} \int_{\Omega} u \mathrm{~d} x \quad \text { and } \quad \tilde{v}=\frac{1}{|\Omega|} \int_{\Omega} v \mathrm{~d} x
$$


The following non-existence conclusion is obtained by mainly considering the self- and cross-diffusion rates. Through a lot of analysis and calculation, we give the following theorem.

Theorem 3.1. Let $C_{1}$ and $M$ be the same as that of in (2.2) and $d_{1}, d_{2}$ satisfy

$$
d_{1}>\mu_{1}^{-1} g(0)+c K_{0}, \quad d_{2}>\mu_{1}^{-1}\left(\frac{c K_{0}+C_{1}^{2}}{4 \mu_{1}}-d\right) .
$$

Denote

$$
D_{3} \triangleq 2 \sqrt{\frac{\mu_{1} d_{1}-g(0)-c \mu_{1} K_{0}}{2 \mu_{1}^{2} d_{1}^{2}+M^{2} S^{2}}}, \quad D_{4} \triangleq \frac{\sqrt{\mu_{1} d_{2}+d-\frac{c K_{0}+\left(1+d_{3} M\right)^{2} S^{2}}{4}}}{4 \mu_{1}}
$$

with $K_{0}=\sup _{\bar{\Omega}} \tilde{v} p^{\prime}(u)$. Then system (1.2) has no non-constant positive solution when $d_{3}<D_{3}$ and $d_{4}<D_{4}$.

Proof. Suppose that $(u, v)$ is a positive solution of system (1.2). Multiplying $u-\tilde{u}$ and $v-\tilde{v}$ to the equations of system (1.2), respectively, then integrating the equation over $\Omega$, we obtain

$$
\begin{aligned}
& \int_{\Omega}\left\{(u-\tilde{u}) d_{1} \Delta\left[\left(1+d_{3} v\right) u\right]+(v-\tilde{v}) d_{2} \Delta\left[\left(1+d_{4} u\right) v\right]\right\} \mathrm{d} x \\
= & \int_{\Omega}\left[d_{1}\left(1+d_{3} v\right)|\nabla u|^{2}+d_{1} d_{3} u \nabla u \nabla v+d_{2}\left(1+d_{4} u\right)|\nabla v|^{2}+d_{2} d_{4} v \nabla u \nabla v\right] \mathrm{d} x \\
= & \int_{\Omega}[(u-\tilde{u})(u g(u)-p(u) v)+(v-\tilde{v}) v(-d+c p(u))] \mathrm{d} x \\
= & \int_{\Omega}(u-\tilde{u})[g(u)(u-\tilde{u})+\tilde{u}(g(u)-g(\tilde{u}))-p(u)(v-\tilde{v})-\tilde{v}(p(u)-p(\tilde{u}))] \mathrm{d} x \\
& +\int_{\Omega}(v-\tilde{v})[-d(v-\tilde{v})+c p(u)(v-\tilde{v})+c \tilde{v}(p(u)-p(\tilde{u}))] \mathrm{d} x \\
= & \left.\int_{\Omega}(u-\tilde{u})\left[g(u)(u-\tilde{u})+\tilde{u} g^{\prime}(\xi)(u-\tilde{u})-p(u)(v-\tilde{v})-\tilde{v} p^{\prime}(\eta)(u-\tilde{u})\right)\right] \mathrm{d} x \\
& +\int_{\Omega}(v-\tilde{v})\left[-d(v-\tilde{v})+c p(u)(v-\tilde{v})+c \tilde{v}(u-\tilde{u}) p^{\prime}(\zeta)\right] \mathrm{d} x \\
= & \int_{\Omega}\left\{(u-\tilde{u})^{2}\left[g(u)+\tilde{u} g^{\prime}(\xi)-\tilde{v} p^{\prime}(\eta)\right]+(v-\tilde{v})^{2}[-d+c p(u)]+(u-\tilde{u})(v-\tilde{v})\left[-p(u)+c \tilde{v} p^{\prime}(\zeta)\right]\right\} \mathrm{d} x \\
\leq & \int_{\Omega}\left[(u-\tilde{u})^{2} g(0)+(v-\tilde{v})^{2}\left(-d+c p\left(C_{1}\right)\right)+c K_{0}|u-\tilde{u} \| v-\tilde{v}|\right] \mathrm{d} x,
\end{aligned}
$$

where $\xi, \eta, \zeta$ are positive constants satisfying $0<\xi, \eta, \zeta \leq C_{1}$ and $K_{0}=\sup _{\bar{\Omega}} \tilde{v} p^{\prime}(u)$. By the Cauchy inequality and Theorem 2.3, we have

$$
\begin{aligned}
\int_{\Omega}\left[d_{1}|\nabla u|^{2}+d_{2}|\nabla v|^{2}\right] \mathrm{d} x \leq & \int_{\Omega}\left[d_{1}\left(1+d_{3} v\right)|\nabla u|^{2}+d_{2}\left(1+d_{4} u\right)|\nabla v|^{2}\right] \mathrm{d} x \\
\leq & \int_{\Omega}\left[(u-\tilde{u})^{2}\left(g(0)+c K_{0} K\right)+(v-\tilde{v})^{2}\left(-d+c p\left(C_{1}\right)+\frac{c K_{0}}{4 K}\right)\right. \\
& \left.+K d_{1}^{2} d_{3}^{2}|\nabla u|^{2}+\frac{C_{1}^{2}}{4 K}|\nabla v|^{2}+K d_{2}^{2} d_{4}^{2}|\nabla v|^{2}+\frac{C_{1}^{2}}{4 K}|\nabla u|^{2}\right] \mathrm{d} x
\end{aligned}
$$


with $K$ be an arbitrary positive constant. We obtain the inequality from the fact

$$
c K_{0}|u-\tilde{u}||v-\tilde{v}|=2 \sqrt{c K_{0} K}|u-\tilde{u}| \sqrt{\frac{c K_{0}}{4 K}}|v-\tilde{v}| \leq c K_{0} K(u-\tilde{u})^{2}+\frac{c K_{0}}{4 K}(v-\tilde{v})^{2} .
$$

Particularly, taking $K=\mu_{1}$ and using the well-known Poincaré inequality, we have

$$
\begin{aligned}
& \int_{\Omega}\left[d_{1}|\nabla u|^{2}+d_{2}|\nabla v|^{2}\right] \mathrm{d} x \\
\leq & \int_{\Omega}\left[\mu_{1}^{-1}\left(g(0)+c K_{0} \mu_{1}+\mu_{1}^{2} d_{1}^{2} d_{3}^{2}+\frac{C_{1}^{2}}{4}\right)\right]|\nabla u|^{2} \mathrm{~d} x \\
& +\int_{\Omega}\left[\mu_{1}^{-1}\left(-d+c p\left(C_{1}\right)+\frac{c K_{0}}{4 \mu_{1}}+\mu_{1}^{2} d_{2}^{2} d_{4}^{2}+\frac{C_{1}^{2}}{4}\right)\right]|\nabla v|^{2} \mathrm{~d} x .
\end{aligned}
$$

Recall that $C_{1}=\left(1+d_{3} M\right) S$ is given in (2.2) and, by direct computation, we know that system (1.2) has no non-constant positive solutions when

$$
d_{1}>\mu_{1}^{-1}\left(g(0)+c K_{0} \mu_{1}+\mu_{1}^{2} d_{1}^{2} d_{3}^{2}+\frac{C_{1}^{2}}{4}\right) \quad \text { and } \quad d_{2}>\mu_{1}^{-1}\left(-d+c p\left(C_{1}\right)+\frac{c K_{0}}{4 \mu_{1}}+\mu_{1}^{2} d_{2}^{2} d_{4}^{2}+\frac{C_{1}^{2}}{4}\right),
$$

i.e.,

$$
d_{3}<2 \sqrt{\frac{\mu_{1} d_{1}-g(0)-c \mu_{1} K_{0}}{2 \mu_{1}^{2} d_{1}^{2}+M^{2} S^{2}}} \triangleq D_{3} \quad \text { and } \quad d_{4}<\frac{\sqrt{\mu_{1} d_{2}+d-\frac{c K_{0}+\left(1+d_{3} M\right)^{2} S^{2}}{4}}}{4 \mu_{1}} \triangleq D_{4}
$$

Thus, the proof is completed.

Remark 3.2. If $d_{3}$ and $d_{4}$ are small enough, say $d_{3} \leq d_{3}^{*}, d_{4} \leq d_{4}^{*}$ for some positive constant $d_{3}^{*}, d_{4}^{*}$, then Theorem 3.1 shows that system (1.2) does not admit a non-constant positive solution for some large enough diffusions $d_{1}, d_{2}$. This is consistent with the the result in $[8,13]$.

\section{EXISTENCE AND STABILITY OF THE NON-CONSTANT POSITIVE SOLUTiOnS}

In this part, we mainly discuss the existence of non-constant positive solutions to system (1.2) by using the self- and cross- diffusion coefficients as parameters. In particular, we focus on the cases that the cross-diffusion is small enough and the self-diffusion is large enough. The key method used here in proving the existence of non-constant positive solutions is the well-known Leray-Schauder degree theory [10], which has been extensively used in many different articles. Moreover, we also discuss the Turing instability of system (1.1) mainly by considering the influence of the diffusion terms.

\subsection{Local analysis at the constant positive solution}

In this subsection, we discuss the linearization of system (1.2) at the constant positive equilibrium point $\boldsymbol{W}^{*}=\left(u^{*}, v^{*}\right)^{T}$. In order to facilitate, we need introduce some frequently-used definitions and notations in advance.

Let $\left\{\mu_{i}, \psi_{i}\right\}_{i=0}^{\infty}$ be a complete set of eigenpairs for the operator $-\Delta$ in $\Omega$ under homogeneous Neumann boundary condition. Set

$$
\boldsymbol{X}=\left\{(u, v)^{T} \in\left(C^{2}(\Omega) \cap C^{1}(\bar{\Omega})\right)^{2} \mid \partial_{\nu} u=\partial_{\nu} v=0 \text { on } \partial \Omega\right\}
$$


We decompose $\boldsymbol{X}=\oplus_{i=0}^{\infty} \boldsymbol{X}_{i}$, where $\boldsymbol{X}_{i}$ is the eigenspace corresponding to the eigenvalue $\mu_{i}$. Let

$$
\begin{gathered}
\Lambda=\left(d_{1}, d_{2}, d_{3}, d_{4}\right), \quad \boldsymbol{B}(C)=\left\{(u, v)^{T} \in \boldsymbol{X} \mid C^{-1}<u, v<C\right\}, \\
\boldsymbol{W}=(u, v)^{T}, \quad \boldsymbol{\Phi}(\boldsymbol{W})=\left(d_{1}\left(1+d_{3} v\right) u, d_{2}\left(1+d_{4} u\right) v\right)^{T}
\end{gathered}
$$

and

$$
\boldsymbol{G}(\boldsymbol{W})=(u g(u)-p(u) v, v(-d+c p(u)))^{T} .
$$

By direct computation, we know that system (1.2) only has a unique constant positive equilibrium point $\boldsymbol{W}^{*}=$ $\left(u^{*}, v^{*}\right)^{T}$ when the assumptions (A1) and (A2) hold, where

$$
u^{*}=p^{-1}\left(\frac{d}{c}\right), \quad v^{*}=\frac{u^{*} g\left(u^{*}\right)}{p\left(u^{*}\right)}
$$

with $p^{-1}(u)$ being the inverse of the function $p(u)$.

Since $\frac{\partial \boldsymbol{\Phi}(u, v)}{\partial(u, v)}=\left[\begin{array}{cc}d_{1}+d_{1} d_{3} v & d_{1} d_{3} u \\ d_{2} d_{4} v & d_{2}+d_{2} d_{4} u\end{array}\right]$ and $\operatorname{det}\left[\frac{\partial \Phi(u, v)}{\partial(u, v)}\right]>0$ for all non-negative solutions $(u, v)^{T}$, so $\boldsymbol{\Phi}_{W}^{-1}$ exists and $\operatorname{det}\left[\frac{\partial \boldsymbol{\Phi}(u, v)}{\partial(u, v)}\right]^{-1}$ is positive. Therefore, we can rewrite system (1.2) as the form

$$
\boldsymbol{F}(\Lambda ; \boldsymbol{W}) \triangleq \boldsymbol{W}-(\boldsymbol{I}-\Delta)^{-1}\left\{\boldsymbol{\Phi}_{\boldsymbol{W}}^{-1}(\boldsymbol{W})\left[\boldsymbol{G}(\boldsymbol{W})+\nabla \boldsymbol{W} \Phi_{\boldsymbol{W} \boldsymbol{W}}(\boldsymbol{W}) \nabla \boldsymbol{W}\right]+\boldsymbol{W}\right\}=0,
$$

where $\boldsymbol{I}$ is the identity and $(\boldsymbol{I}-\Delta)^{-1}$ is the inverse of the operator $\boldsymbol{I}-\Delta$ on $\boldsymbol{X}$ under the homogeneous Neumann boundary condition. Since $\boldsymbol{F}(\Lambda ; \cdot)$ is a compact perturbation of the identity operator, the Leray-Schauder degree $\operatorname{deg}(\boldsymbol{F}(\Lambda ; \cdot), \mathbf{0}, \boldsymbol{B})$ is well-defined if $\boldsymbol{F}(\Lambda ; \boldsymbol{W}) \neq 0$ for all $\boldsymbol{W} \in \partial \boldsymbol{B}$.

The linearizition of the operator $\boldsymbol{F}(\Lambda ; \boldsymbol{W})$ at the equilibrium point $\boldsymbol{W}^{*}$ is

$$
D_{\boldsymbol{W}} \boldsymbol{F}\left(\Lambda ; \boldsymbol{W}^{*}\right)=\boldsymbol{I}-(\boldsymbol{I}-\Delta)^{-1}\left[\boldsymbol{\Phi}_{\boldsymbol{W}}^{-1}\left(\boldsymbol{W}^{*}\right) \boldsymbol{G}_{\boldsymbol{W}}\left(\boldsymbol{W}^{*}\right)+\boldsymbol{I}\right] .
$$

It is easy to know that $\boldsymbol{X}_{i}$ is invariant under $D_{\boldsymbol{W}} \boldsymbol{F}\left(\Lambda ; \boldsymbol{W}^{*}\right)$ for each integer $i \geq 0$. Furthermore, $\lambda$ is an eigenvalue of the operator $D_{\boldsymbol{W}} \boldsymbol{F}\left(\Lambda ; \boldsymbol{W}^{*}\right)$ on $\boldsymbol{X}_{i}$ if and only if $\lambda$ is an eigenvalue of the matrix

$$
\boldsymbol{I}-\frac{1}{1+\mu_{i}}\left[\boldsymbol{\Phi}_{\boldsymbol{W}}^{-1}\left(\boldsymbol{W}^{*}\right) \boldsymbol{G}_{\boldsymbol{W}}\left(\boldsymbol{W}^{*}\right)+\boldsymbol{I}\right]=\frac{1}{1+\mu_{i}}\left[\mu_{i} \boldsymbol{I}-\boldsymbol{\Phi}_{\boldsymbol{W}}{ }^{-1}\left(\boldsymbol{W}^{*}\right) \boldsymbol{G}_{\boldsymbol{W}}\left(\boldsymbol{W}^{*}\right)\right] .
$$

Let

$$
H\left(\Lambda, \boldsymbol{W}^{*} ; \mu_{i}\right) \triangleq \operatorname{det}\left[\mu_{i} \boldsymbol{I}-\boldsymbol{\Phi}_{\boldsymbol{W}}^{-1}\left(\boldsymbol{W}^{*}\right) \boldsymbol{G}_{\boldsymbol{W}}\left(\boldsymbol{W}^{*}\right)\right]=\operatorname{det}\left\{\left[\boldsymbol{\Phi}_{\boldsymbol{W}}\left(\boldsymbol{W}^{*}\right)\right]^{-1}\right\} \operatorname{det}\left[\mu \boldsymbol{\Phi}_{\boldsymbol{W}}\left(\boldsymbol{W}^{*}\right)-\boldsymbol{G}_{\boldsymbol{W}}\left(\boldsymbol{W}^{*}\right)\right] .
$$

Since the sign of the

$$
\operatorname{det}\left\{\boldsymbol{I}-\frac{1}{1+\mu_{i}}\left[\boldsymbol{\Phi}_{\boldsymbol{W}}^{-1}\left(\boldsymbol{W}^{*}\right) \boldsymbol{G}_{\boldsymbol{W}}\left(\boldsymbol{W}^{*}\right)+\boldsymbol{I}\right]\right\}
$$

depends on the number of negative eigenvalue of the matrix

$$
\boldsymbol{I}-\frac{1}{1+\mu_{i}}\left[\boldsymbol{\Phi}_{\boldsymbol{W}}^{-1}\left(\boldsymbol{W}^{*}\right) \boldsymbol{G}_{\boldsymbol{W}}\left(\boldsymbol{W}^{*}\right)+\boldsymbol{I}\right]
$$


both $H\left(\Lambda, \boldsymbol{W}^{*} ; \mu_{i}\right)$ and $\operatorname{det}\left\{\boldsymbol{I}-\frac{1}{1+\mu_{i}}\left[\boldsymbol{\Phi}_{\boldsymbol{W}^{-1}}\left(\boldsymbol{W}^{*}\right) \boldsymbol{G}_{\boldsymbol{W}}\left(\boldsymbol{W}^{*}\right)+\boldsymbol{I}\right]\right\}$ have the same signs. Therefore, if $H\left(\Lambda, \boldsymbol{W}^{*} ; \mu_{i}\right) \neq 0$, then the number of eigenvalues with negative real parts of $\boldsymbol{D}_{\boldsymbol{W}} \boldsymbol{F}\left(\Lambda ; \boldsymbol{W}^{*}\right)$ on $\boldsymbol{X}_{\boldsymbol{i}}$ is odd if and only if $H\left(\Lambda, \boldsymbol{W}^{*} ; \lambda_{i}\right)<0$.

If $H\left(\Lambda, \boldsymbol{W}^{*} ; \mu_{i}\right) \neq 0$ for all integer $i \geq 0$, then 0 is not an eigenvalue of the operator $\boldsymbol{D}_{\boldsymbol{W}} \boldsymbol{F}\left(\Lambda ; \boldsymbol{W}^{*}\right)$. This implies that $\boldsymbol{D}_{\boldsymbol{W}} \boldsymbol{F}\left(\Lambda ; \boldsymbol{W}^{*}\right)$ is a homeomorphism operator from the space $\boldsymbol{X}$ to $\boldsymbol{X}$. Then the implicit function theorem shows that the equilibrium point $\boldsymbol{W}=\boldsymbol{W}^{*}$ is an isolated solution of equation $\boldsymbol{F}(\Lambda ; \boldsymbol{W})=0$. In conclusion, according to Leray-Schauder degree theory, we give the following results (One can refer to [19]).

Lemma 4.1. Assume that, for each $i>0$, the matrix $\mu_{i} \boldsymbol{I}-\boldsymbol{\Phi}_{\boldsymbol{W}}{ }^{-1}\left(\boldsymbol{W}^{*}\right) \boldsymbol{G}_{\boldsymbol{W}}\left(\boldsymbol{W}^{*}\right)$ is non-singular. Then

$$
\operatorname{index}\left(\boldsymbol{F}(\Lambda ; \cdot), \boldsymbol{W}^{*}\right)=(-1)^{\sigma}, \quad \sigma=\sum_{i \geq 0, H\left(D, \boldsymbol{W}^{*} ; \mu_{i}\right)<0} \operatorname{dim} \boldsymbol{E}\left(\mu_{i}\right) .
$$

In order to finish the computation of index $\left(\boldsymbol{F}(\Lambda ; \cdot), \boldsymbol{W}^{*}\right)$, we will discuss the sign of $H\left(\Lambda, \boldsymbol{W}^{*} ; \mu_{i}\right)$ thoroughly. Note that $\operatorname{det}\left\{\boldsymbol{\Phi}_{\boldsymbol{W}}\left(\boldsymbol{W}^{*}\right)^{-1}\right\}>0$, so we only need to study $\operatorname{det}\left[\mu \boldsymbol{\Phi}_{\boldsymbol{W}}\left(\boldsymbol{W}^{*}\right)-\boldsymbol{G}_{\boldsymbol{W}}\left(\boldsymbol{W}^{*}\right)\right]$. Recall that $u^{*}=$ $p^{-1}\left(\frac{d}{c}\right)$, and by direct computation, we have

$$
\begin{gathered}
\boldsymbol{\Phi}_{\boldsymbol{W}}\left(\boldsymbol{W}^{*}\right)=\left[\begin{array}{cc}
d_{1}\left(1+d_{3} v^{*}\right) & d_{1} d_{3} u^{*} \\
d_{2} d_{4} v^{*} & d_{2}\left(1+d_{4} u^{*}\right)
\end{array}\right], \\
\boldsymbol{G}_{\boldsymbol{W}}\left(\boldsymbol{W}^{*}\right)=\left[\begin{array}{cc}
g\left(u^{*}\right)+u^{*} g_{u}\left(u^{*}\right)-p_{u}\left(u^{*}\right) v^{*} & -p\left(u^{*}\right) \\
c v^{*} p_{u}\left(u^{*}\right) & 0
\end{array}\right],
\end{gathered}
$$

and

$$
\operatorname{det}\left[\mu \boldsymbol{\Phi}_{\boldsymbol{W}}\left(\boldsymbol{W}^{*}\right)-\boldsymbol{G}_{\boldsymbol{W}}\left(\boldsymbol{W}^{*}\right)\right]=A \mu^{2}+B \mu+C,
$$

where

$$
\begin{gathered}
A=d_{1} d_{2}\left(1+d_{4} u^{*}+d_{3} v^{*}\right)>0, \quad C=c v^{*} p_{u}\left(u^{*}\right) p\left(u^{*}\right), \\
B=\left(d_{2}+d_{2} d_{4} u^{*}\right)\left[g\left(u^{*}\right)+u^{*} g_{u}\left(u^{*}\right)-p_{u}\left(u^{*}\right) v^{*}\right]+c d_{1} d_{3} u^{*} v^{*} p_{u}\left(u^{*}\right)-d_{2} d_{4} v^{*} p\left(u^{*}\right) .
\end{gathered}
$$

When $B^{2}-4 A C>0$, the function $A \mu^{2}+B \mu+C=0$ has two roots denoted by $\mu_{1}(\Lambda), \mu_{2}(\Lambda)$, respectively, with $\mu_{1}(\Lambda)<\mu_{2}(\Lambda)$, where

$$
\mu_{1}(\Lambda)=\frac{-B-\sqrt{B^{2}-4 A C}}{2 A}=\frac{2 C}{-B+\sqrt{B^{2}-4 A C}}, \quad \mu_{2}(\Lambda)=\frac{-B+\sqrt{B^{2}-4 A C}}{2 A}=\frac{2 C}{-B-\sqrt{B^{2}-4 A C}} .
$$

In fact, if $g\left(u^{*}\right)+u^{*} g_{u}\left(u^{*}\right)-p_{u}\left(u^{*}\right) v^{*}>0$ be large, then we can achieve the result. Note that $B>0$ when $g\left(u^{*}\right)+$ $u^{*} g_{u}\left(u^{*}\right)-p_{u}\left(u^{*}\right) v^{*}>0$ and $c d_{1} d_{3} u^{*} v^{*} p_{u}\left(u^{*}\right)-d_{2} d_{4} v^{*} p\left(u^{*}\right)>0$, whereas $B<0$ when $g\left(u^{*}\right)+u^{*} g_{u}\left(u^{*}\right)-$ $p_{u}\left(u^{*}\right) v^{*}<0$ and $c d_{1} d_{3} u^{*} v^{*} p_{u}\left(u^{*}\right)-d_{2} d_{4} v^{*} p\left(u^{*}\right)<0$. In conclusion, we have the following lemma.

Lemma 4.2. Assume that $d_{1}>0, d_{2}>0, d_{3} \geq 0$ and $d_{4} \geq 0$ such that $B^{2}-4 A C>0$. Then

(i) If $g\left(u^{*}\right)+u^{*} g_{u}\left(u^{*}\right)-p_{u}\left(u^{*}\right) v^{*}>0$ and $c d_{1} d_{3} u^{*} v^{*} p_{u}\left(u^{*}\right)-d_{2} d_{4} v^{*} p\left(u^{*}\right)>0$, then $B>0$ and both the roots $\mu_{1}(\Lambda)$ and $\mu_{2}(\Lambda)$ are negative.

(ii) If $g\left(u^{*}\right)+u^{*} g_{u}\left(u^{*}\right)-p_{u}\left(u^{*}\right) v^{*}<0$ and $c d_{1} d_{3} u^{*} v^{*} p_{u}\left(u^{*}\right)-d_{2} d_{4} v^{*} p\left(u^{*}\right)<0$, then $B<0$ and both the roots $\mu_{1}(\Lambda)$ and $\mu_{2}(\Lambda)$ are positive. 
Now, we investigate the dependence of $\mu_{1}(\Lambda)$ and $\mu_{2}(\Lambda)$ on $d_{i}, i=1,2,3,4$ in the case of $B<0$. By direct computation, we have the following result.

Lemma 4.3. Assume that $d_{1}>0, d_{2}>0, d_{3} \geq 0$ and $d_{4} \geq 0$ such that $B^{2}-4 A C>0$. Then, we have the followings.

(i) Let $d_{2}>0, d_{3} \geq 0$ and $d_{4} \geq 0$ be fixed. Then

$$
\lim _{d_{1} \rightarrow 0^{+}} \mu_{1}(\Lambda)=\lim _{d_{1} \rightarrow 0^{+}} \frac{2 C}{-B+\sqrt{B^{2}-4 A C}}=\frac{c v^{*} p_{u}\left(u^{*}\right) p\left(u^{*}\right)}{\left(d_{2}+d_{2} d_{4} u^{*}\right)\left[g\left(u^{*}\right)+u^{*} g_{u}\left(u^{*}\right)-p_{u}\left(u^{*}\right) v^{*}\right]-d_{2} d_{4} v^{*} p\left(u^{*}\right)},
$$

$$
\lim _{d_{1} \rightarrow 0^{+}} \mu_{2}(\Lambda)=\lim _{d_{1} \rightarrow 0^{+}} \frac{2 C}{-B-\sqrt{B^{2}-4 A C}}=+\infty
$$

where $\mu_{1}(\Lambda)>0$ provided

$$
d_{4}>\frac{g\left(u^{*}\right)+u^{*} g_{u}\left(u^{*}\right)-p_{u}\left(u^{*}\right) v^{*}}{g\left(u^{*}\right)+u^{*} g_{u}\left(u^{*}\right)-\left[p_{u}\left(u^{*}\right)+p\left(u^{*}\right)\right] v^{*}} .
$$

(ii) Let $d_{1}>0, d_{3} \geq 0$ and $d_{4} \geq 0$ be fixed. Then, we have

$$
\lim _{d_{2} \rightarrow 0^{+}} \mu_{1}(\Lambda)=\lim _{d_{2} \rightarrow 0^{+}} \frac{2 C}{-B+\sqrt{B^{2}-4 A C}}=\frac{p\left(u^{*}\right)}{d_{1} d_{3} u^{*}}, \quad \lim _{d_{2} \rightarrow 0^{+}} \mu_{2}(\Lambda)=\lim _{d_{2} \rightarrow 0^{+}} \frac{2 C}{-B-\sqrt{B^{2}-4 A C}}=+\infty .
$$

Moreover, $0<\lim _{d_{2} \rightarrow 0^{+}} \mu_{1}(\Lambda)<\mu_{1}$ when one of $d_{1}$ and $d_{3}$ is large enough.

Furthermore, combining with (4.2), we obtain the following lemma.

Lemma 4.4. If $0<\mu_{1}(\Lambda)<\mu_{i}<\mu_{2}(\Lambda)$ for some $i \geq 0$, then $H\left(\mu_{i}\right)<0$. Whereas, if $\mu_{i} \notin\left[\mu_{1}(\Lambda), \mu_{2}(\Lambda)\right]$, then $H\left(\mu_{i}\right)>0$ for some $i \geq 0$.

\subsection{Existence of the non-constant positive solutions}

In the following, by using Leray-Schauder degree theory, we investigate the existence of non-constant positive solutions to system (1.2) with respect to the cross coefficients $d_{i}, i=1,2,3,4$. In view of Lemma 4.3 , we can obtain the following theorem.

Theorem 4.5. Assume that $d_{1}>0, d_{2}>0, d_{3} \geq 0$ and $d_{4} \geq 0$ satisfying $B^{2}-4 A C>0$ and let $d_{4}$ be fixed.

(i) Let $D_{1}\left(d_{2}, d_{3}, d_{4}\right)$ be a positive constants satisfying Lemma 4.3 (ii). For $d_{1}>D_{1}\left(d_{2}, d_{3}, d_{4}\right)$, if there exists some integer $n \geq 1$ such that $\mu_{1}(\Lambda) \in\left(\mu_{0}, \mu_{1}\right), \mu_{2}(\Lambda) \in\left(\mu_{n}, \mu_{n+1}\right)$ and the sum $\sigma_{n}=\sum_{1}^{n} \operatorname{dim} E\left(\mu_{i}\right)$ is odd, then system (1.2) has non-constant positive solutions.

(ii) Let $D_{3}\left(d_{1}, d_{2}, d_{4}\right)$ be a positive constants satisfying Lemma 4.3 (ii). For $d_{3}>D_{3}\left(d_{1}, d_{2}\right.$, $\left.d_{4}\right)$, if there exists some integer $n \geq 1$ such that $\mu_{1}(\Lambda) \in\left(\mu_{0}, \mu_{1}\right), \mu_{2}(\Lambda) \in\left(\mu_{n}, \mu_{n+1}\right)$ and the sum $\sigma_{n}=\sum_{1}^{n} \operatorname{dim} E\left(\mu_{i}\right)$ is odd, then system (1.2) has non-constant positive solutions.

Proof. We only prove the first assertion and the second result can be proved similarly. From Theorem 2.3 and Lemma 4.3, we know that there exist positive constants $D_{1}=D_{1}\left(d_{2}, d_{3}, d_{4}\right)$ such that $\mu_{1}(\Lambda) \in\left(\mu_{0}, \mu_{1}\right), \mu_{2}(\Lambda) \in$ $\left(\mu_{n}, \mu_{n+1}\right)$. By Theorem 3.1, we know that there exist large $d_{1}^{*}, d_{2}^{*}$ and small $d_{3}, d_{4}$ such that (1.2) does not permit nonconstant positive solution. Denote $\Lambda^{*}=\left(d_{1}^{*}, d_{2}^{*}, d_{3}, d_{4}\right)$. 
For $\tau \in[0,1]$, we define a homotopy as

$$
\begin{cases}-\Delta\left[\tau d_{1}+(1-\tau) d_{1}^{*}+\tau d_{1} d_{3} v\right] u=u g(u)-p(u) v, & x \in \Omega, \\ -\Delta\left[\tau d_{2}+(1-\tau) d_{2}^{*}+\tau d_{2} d_{4} u\right] v=v(-d+c p(u)), & x \in \Omega, \\ \partial_{\nu} u=\partial_{\nu} v=0, & x \in \partial \Omega\end{cases}
$$

Denote

$$
\boldsymbol{\Phi}(\tau, \boldsymbol{W})=\left(\left[\tau d_{1}+(1-\tau) d_{1}^{*}+\tau d_{1} d_{3} v\right] u,\left[\tau d_{2}+(1-\tau) d_{2}^{*}+\tau d_{2} d_{4} u\right] v\right)^{T} .
$$

Therefore, system (4.3) can be rewritten as

$$
\begin{cases}-\Delta \boldsymbol{\Phi}(\tau, \boldsymbol{W})=\boldsymbol{G}(\boldsymbol{W}), & x \in \Omega \\ \partial \boldsymbol{\Phi}(\tau, \boldsymbol{W})=0, & x \in \partial \Omega\end{cases}
$$

Then $\boldsymbol{W}$ is a non-constant positive solution of system (1.2) if and only if $\boldsymbol{W}$ is a non-constant positive solution of (4.4) for $\tau=1$. Therefore, for any $\tau \in[0,1], \boldsymbol{W}$ is a positive solution of system (4.4) if and only if

$$
\widetilde{\boldsymbol{F}}(\tau, \Lambda ; \boldsymbol{W}) \triangleq \boldsymbol{W}-(\boldsymbol{I}-\Delta)^{-1}\left\{\widetilde{\boldsymbol{\Phi}}_{\boldsymbol{W}}{ }^{-1}(\tau, \boldsymbol{W})\left[\boldsymbol{G}(\boldsymbol{W})+\nabla \boldsymbol{W} \widetilde{\boldsymbol{\Phi}}_{\boldsymbol{W} \boldsymbol{W}}(\tau, \boldsymbol{W}) \boldsymbol{\nabla} \boldsymbol{W}\right]+\boldsymbol{W}\right\}=0, \boldsymbol{W} \in \boldsymbol{X} .
$$

By a direct computation, we obtain

$$
\boldsymbol{D}_{\boldsymbol{W}} \widetilde{\boldsymbol{F}}\left(\tau, \Lambda ; \boldsymbol{W}^{*}\right)=\boldsymbol{I}-(\boldsymbol{I}-\Delta)^{-1}\left[\boldsymbol{\Phi}_{\boldsymbol{W}^{-1}}\left(\tau ; \boldsymbol{W}^{*}\right) \boldsymbol{G}_{\boldsymbol{W}}\left(\boldsymbol{W}^{*}\right)+\boldsymbol{I}\right] .
$$

For $\tau=1$, making use of Lemma 4.4, we have $H\left(\mu_{0}\right)>0, H\left(\mu_{i}\right)<0$ for $1 \leq i \leq n, H\left(\mu_{i}\right)>0$ for $i>n$, where $H(\mu)$ defined as before. Therefore, we have

$$
\sum_{H\left(\mu_{i}\right)<0} \operatorname{dim} \boldsymbol{E}\left(\mu_{i}\right)=\sum_{i=1}^{n} \operatorname{dim} \boldsymbol{E}\left(\mu_{i}\right)=\sigma_{n}
$$

Suppose, on the contrary, that system (1.2) has no non-constant positive solution. Since $\sigma$ is odd, by Lemma 4.1, we obtain

$$
\operatorname{index}\left(\boldsymbol{F}(\Lambda ; \cdot), \boldsymbol{W}^{*}\right)=-1
$$

According to Theorem 2.3, it is easy to know that all the positive solutions $(u, v)$ of system $(1.2)$ are in $\boldsymbol{B}(C)$ for some large enough constant $C$. Therefore, for all $\tau \in[0,1]$, system (4.4) has no positive solutions on $\partial \boldsymbol{B}$, and $\operatorname{deg}(\widetilde{\boldsymbol{F}}(\tau, \Lambda ; \cdot), 0, \boldsymbol{B})$ is well defined. According to the homotopy invariance theory of the Leray-Schauder degree, we obtain

$$
\operatorname{deg}(\widetilde{\boldsymbol{F}}(0, \Lambda ; \cdot), 0, \boldsymbol{B})=\operatorname{deg}(\widetilde{\boldsymbol{F}}(1, \Lambda ; \cdot), 0, \boldsymbol{B})
$$

On the other hand,

$$
\boldsymbol{\Phi}(\boldsymbol{W})=\widetilde{\boldsymbol{\Phi}}(1, \boldsymbol{W}), \quad \boldsymbol{F}(\Lambda ; \boldsymbol{W})=\widetilde{\boldsymbol{F}}(1, \Lambda ; \boldsymbol{W}), \quad \boldsymbol{F}\left(\Lambda^{*} ; \boldsymbol{W}\right)=\widetilde{\boldsymbol{F}}(0, \Lambda ; \boldsymbol{W}),
$$

and

$$
\operatorname{deg}(\widetilde{\boldsymbol{F}}(0, \Lambda ; \cdot), 0, \boldsymbol{B})=\operatorname{deg}\left(\boldsymbol{F}\left(\Lambda^{*} ; \cdot\right), 0, \boldsymbol{B}\right),
$$




$$
\operatorname{deg}(\widetilde{\boldsymbol{F}}(1, \Lambda ; \cdot), 0, \boldsymbol{B})=\operatorname{deg}(\boldsymbol{F}(\Lambda ; \cdot), 0, \boldsymbol{B})
$$

Furthermore, if system (1.2) has no non-constant positive solution, we have shown

$$
\operatorname{deg}\left(\boldsymbol{F}\left(\Lambda^{*} ; \cdot\right), 0, \boldsymbol{B}\right)=1
$$

Therefore, we have

$$
\operatorname{deg}(\boldsymbol{F}(\Lambda ; \cdot), 0, \boldsymbol{B})=\operatorname{deg}\left(\boldsymbol{F}\left(\Lambda^{*} ; \cdot\right), 0, \boldsymbol{B}\right)=1
$$

By (4.5)-(4.6), we obtain a contradiction. Hence, $\boldsymbol{F}(\Lambda ; \boldsymbol{W})=0$ has at least one positive solution which is different from the constant solution, namely, system (1.2) has at least one non-constant positive solution and we complete the proof.

\subsection{Turing instability of the positive constant solution}

In this subsection, we focus on the stability of the positive constant solution $\boldsymbol{W}^{*}=\left(u^{*}, v^{*}\right)^{T}$. Using the notations in subsection 4.1, it is easy to know that system (1.1) can be written as

$$
\begin{cases}\boldsymbol{W}_{t}-\Delta \boldsymbol{\Phi}(\boldsymbol{W})=\boldsymbol{G}(\boldsymbol{W}), & (x, t) \in \Omega \times(0, \infty) \\ \partial_{\nu} \boldsymbol{W}=0, & (x, t) \in \partial \Omega \times(0, \infty) \\ \boldsymbol{W}(x, 0)=\left(u_{0}(x), v_{0}(x)\right)^{T}, & x \in \Omega\end{cases}
$$

Thus, the corresponding spatially homogeneous counterpart of system (4.7) is

$$
\boldsymbol{W}_{t}=\boldsymbol{G}(\boldsymbol{W}), \quad t \in(0, \infty) .
$$

The Turing instability means diffusion drives instability. Since the introduction of the self- and cross-diffusion terms, the stability of the positive constant solutions $\boldsymbol{W}^{*}$ may change from the stable, for the ODE system (4.8), to unstable, for the PDE system (4.7).

In the following, we discuss the stability of the constant positive solution $\boldsymbol{W}^{*}=\left(u^{*}, v^{*}\right)^{T}$ of system (4.8). The linearization of system (4.8) at $\boldsymbol{W}^{*}$ can be expressed by

$$
\boldsymbol{W}_{t}=\boldsymbol{G}_{\boldsymbol{W}}\left(\boldsymbol{W}^{*}\right)
$$

where $\boldsymbol{G}_{\boldsymbol{W}}\left(\boldsymbol{W}^{*}\right)$ is the same as that of given in Subsection 4.1. The character polynomial equation of $\boldsymbol{G}_{\boldsymbol{W}}\left(\boldsymbol{W}^{*}\right)$ is

$$
\lambda^{2}-\left[g\left(u^{*}\right)+u^{*} g_{u}\left(u^{*}\right)-p_{u}\left(u^{*}\right) v^{*}\right] \lambda+c v^{*} p_{u}\left(u^{*}\right) p\left(u^{*}\right)=0 .
$$

In conclusion, we present the following lemma.

Lemma 4.6. Assume that $g\left(u^{*}\right)+u^{*} g_{u}\left(u^{*}\right)-p_{u}\left(u^{*}\right) v^{*}<0$. Then $\operatorname{Re}(\lambda)<0$ and $\boldsymbol{W}^{*}$ is stable.

Next, we investigate the stability of the positive constant solution $\boldsymbol{W}^{*}$ of the PDE dynamics system (4.7). In order to find the criterion for the Turing instability, we first give some calculations.

The linearization of system (4.7) at $\boldsymbol{W}^{*}$ can be written as

$$
\begin{cases}\boldsymbol{W}_{t}-\mathbf{\Phi}_{\boldsymbol{W}}\left(\boldsymbol{W}^{*}\right) \Delta \boldsymbol{W}=\boldsymbol{G}_{\boldsymbol{W}}\left(\boldsymbol{W}^{*}\right) \boldsymbol{W}, & (x, t) \in \Omega \times(0, \infty) \\ \partial_{\nu} W=0, & (x, t) \in \partial \Omega \times(0, \infty) .\end{cases}
$$


We have the following lemma.

Lemma 4.7. If the matrix $-\mu \boldsymbol{\Phi}_{\boldsymbol{W}}\left(\boldsymbol{W}^{*}\right)+\boldsymbol{G}_{\boldsymbol{W}}\left(\boldsymbol{W}^{*}\right)$ has an eigenvalue with positive real part, then the constant positive solution $\boldsymbol{W}^{*}$ of system (4.7) is unstable.

By direct computation, we obtain

$$
\lambda^{2}+\left\{\left(d_{1}+d_{2}+d_{1} d_{3} v^{*}+d_{2} d_{4} u^{*}\right) \mu-\left[g\left(u^{*}\right)+u^{*} g_{u}\left(u^{*}\right)-p_{u}\left(u^{*}\right) v^{*}\right]\right\} \lambda+\operatorname{det}\left[-\mu \boldsymbol{\Phi}_{\boldsymbol{W}}\left(\boldsymbol{W}^{*}\right)+\boldsymbol{G}_{\boldsymbol{W}}\left(\boldsymbol{W}^{*}\right)\right]=0,
$$

with

$$
\operatorname{det}\left[-\mu \boldsymbol{\Phi}_{\boldsymbol{W}}\left(\boldsymbol{W}^{*}\right)+\boldsymbol{G}_{\boldsymbol{W}}\left(\boldsymbol{W}^{*}\right)\right]=\operatorname{det}\left[\mu \boldsymbol{\Phi}_{\boldsymbol{W}}\left(\boldsymbol{W}^{*}\right)-\boldsymbol{G}_{\boldsymbol{W}}\left(\boldsymbol{W}^{*}\right)\right]=A \mu^{2}+B \mu+C,
$$

where $A, B$ and $C$ are given in (4.2).

Combining Lemma 4.1 with Lemma 4.3, we have the following result.

Theorem 4.8. Assume that $d_{1}>0, d_{2}>0, d_{3} \geq 0$ and $d_{4} \geq 0$ such that $B^{2}-4 A C>0$.

(i) Let $d_{2}>0, d_{3} \geq 0$ be fixed. If $d_{4}>\frac{g\left(u^{*}\right)+u^{*} g_{u}\left(u^{*}\right)-p_{u}\left(u^{*}\right) v^{*}}{g\left(u^{*}\right)+u^{*} g_{u}\left(u^{*}\right)-\left[p_{u}\left(u^{*}\right)+p\left(u^{*}\right)\right] v^{*}} \geq 0$, then there exists a positive constant $D_{1}\left(d_{2}, d_{3}, d_{4}\right)$ such that the positive constant solution $\boldsymbol{W}^{*}$ of system (4.7) is unstable when $d_{1}<D_{1}\left(d_{2}, d_{3}, d_{4}\right)$ holds, whereas $\boldsymbol{W}^{*}$ is stable when $d_{1}>D_{1}\left(d_{2}, d_{3}, d_{4}\right)$.

(ii) Let $d_{1}>0, d_{3} \geq 0$ and $d_{4} \geq 0$ be fixed. Then there exists a positive constant $D_{2}\left(d_{1}, d_{3}, d_{4}\right)$ such that the positive constant solution $\boldsymbol{W}^{*}$ of system (4.7) is unstable when $d_{2}<D_{2}\left(d_{1}, d_{3}, d_{4}\right)$ holds, whereas $\boldsymbol{W}^{*}$ is stable when $d_{2}>D_{2}\left(d_{1}, d_{3}, d_{4}\right)$.

Remark 4.9. The result shows that, due to the introduction of diffusion terms, the stability of the positive constant solution $\boldsymbol{W}^{*}$ of the PDE system (4.7) (also (1.1)) may change from stable, for the counterpart ODE system, to unstable under certain conditions.

\section{Comments And CONCLUSIONS}

This paper investigates the existence and non-existence of non-constant positive solutions for a generalized Gause-type predator-prey system with self- and cross-diffusions under homogeneous Neumann boundary condition, in which the cross-diffusions are included in such away that the prey runs away from the predator and the predator moves away from a large group of preys. On the basis of Leray-Schauder degree theory and mathematical analysis, we prove that cross-diffusions can create coexistence for the prey and predator under suitable conditions. Our main results indicate that system (1.2) admits non-constant positive solutions if one of the self-diffusions $d_{1}$ or the cross-diffusions $d_{3}$ is large under certain conditions. Moreover, we also discuss the Turing instability of system (1.1) mainly by considering the influence of the diffusion terms. The results show that the diffusion terms can influence the stability of the system.

The theoretical analysis indicates that the self- and cross-diffusions phenomena have the potential to play an important role for the symbiosis and coexistence of the species. The main distinction between this article and [8] is the introduction of the cross-diffusion factor $d_{4}$. Since we introduce the cross-diffusion $d_{4}$ into the system, compared with [8], the conclusion shows that the coexistence is mainly affected by the self-diffusion rate $d_{1}$ or the cross-diffusion rate $d_{3}$.

Acknowledgements. We are grateful to the anonymous referees and the handling editor for their constructive suggestions and valuable comments on this manuscript.

\section{REFERENCES}

[1] J.H.P. Dawes and M.O. Souza, A derivation of Hollingís type I, II and III functional responses in predator-prey systems. J. Theoret. Biol. 327 (2013) 11-22. 
[2] D.T. Dimitrov and H.V. Kojouharov, Complete mathematical analysis of predator-prey models with linear prey growth and Beddington-DeAngelis. Appl. Math. Comput. 162 (2005) 523-538.

[3] D. Gilbarg and N.S. Trudinger, Elliptic Partial Differential Equation of Second Order. Springer-Verlag, Berlin (2001).

[4] N.S. Goel and S.C. Maitra, On the Volterra and other nonlinear models of interacting populations. Rev. Modern Phys. 43 (1971) 231-276.

[5] Y. Jia, A sufficient and necessary condition for the existence of positive solutions for a prey-predator system with Ivlev-type functional response. Appl. Math. Lett. 24 (2011) 1084-1088.

[6] Y. Jia, J. Wu and H.-K. Xu, Positive solutions for a predator-prey interaction model with Hollings-type functional response and diffusion. Taiwan. J. Math. 15 (2011) 2013-2034.

[7] Y. Jia, J. Wu and H.-K. Xu, Positive solutions of a Lotka-Volterra competition model with cross-diffusion. Comput. Math. Appl. 68 (2014) 1220-1228.

[8] Y. Jia and P. Xue, Effects of the self- and cross-diffusion on positive steady states for a generalized predator-prey system. Nonlinear Anal. Real World Appl. 32 (2016) 229-241.

[9] W. Ko and K. Ryu, A qualitative study on general Gause-type predator-prey models with constant diffusion rates. J. Math. Anal. Appl. 344 (2008) 217-230.

[10] J. Leray and J. Schauder, Topologie et équations fonctionnelles. Ann. Sci. École Norm. Sup. 51 (1934) 45-78.

[11] C.-S. Lin, W.-M. Ni and I. Takagi, Large amplitude stationary solutions to a chemotaxis system. J. Differ. Equ. 72 (1988) $1-27$.

[12] Y. Lou, W.-M. Ni and S. Yotsutani, On a limiting system in the Lotka-Volterra competition with cross-diffusion. Discret. Contin. Dyn. Syst. 10 (2004) 435-458.

[13] Y. Lou and W.-M. Ni, Diffusion, self-diffusion and cross-diffusion. J. Differ. Equ. 131 (1996) 79-131.

[14] A. Madzvamuse, H.S. Ndakwo and R. Barreira, Cross-diffusion-driven instability for reaction-diffusion systems: analysis and simulations. J. Math. Biol. 27 (2014) 262-292.

[15] Q. Meng and L. Yang, Steady state in a cross-diffusion predator-prey model with the Beddington-DeAngelis functional response. Nonlinear Anal. Real World Appl. 45 (2019) 401-413.

[16] W.-M. Ni, Diffusion, cross-diffusion and their spike-layer steady states. Notices Am. Math. Soc. 45 (1998) 9-18.

[17] K. Oeda, Effect of cross-diffusion on the stationary problem of a prey-predator model with a protection zone. J. Differ. Equ. 250 (2011) 3988-4009.

[18] A. Okubo, Diffusion and Ecological Problems: Mathematical Models. Springer-Verlag, Berlin (1980).

[19] P.Y.H. Pang and M. Wang, Non-constant positive steady states of a predator-prey system with non-monotonic functional response and diffusion. Proc. London Math. Soc. 88 (2004) 135-157.

[20] R. Peng and J. Shi, Non-existence of non-constant positive steady states of two Holling-type II predator-prey systems: strong interaction case. J. Differ. Equ. 247 (2009) 866-886.

[21] G. Seo and D.L. DeAngelis, A predator-prey model with a Holling type I functional response including a predator mutual interference. J. Nonlinear Sci. 21 (2011) 811-833.

[22] A.A. Shaikh, H. Das, N. Ali and C. Sonck, Study of LG-Holling type III predator-prey model with disease in predator. J. Appl. Math. Comput. 58 (2018) 235-255.

[23] J. Sugie, Two-parameter bifurcation in a predator-prey system of Ivlev type. J. Math. Anal. Appl. 217 (1998) $349-371$.

[24] J. Shi, Z. Xie and K. Little, Cross-diffusion induced instability and stability in reaction-diffusion systems. J. Appl. Anal. Comput. 1 (2011) 95-119.

[25] X. Zeng, Non-constant positive steady states of a prey-predator system with cross-diffusions. J. Math. Anal. Appl. 332 (2007) 989-1009. 